\title{
A comparison of disease risk analysis tools for conservation translocations
}

\section{Antonia Eleanor Dalziel ${ }^{1,2}$, Anthony W. Sainsbury ${ }^{1}$, Kate McInnes ${ }^{3}$, Richard Jakob-Hoff ${ }^{4}$ and}

John G. Ewen ${ }^{1}$

${ }^{1}$ Institute of Zoology, Zoological Society of London, Regents Park, NW1 4RY, London, United Kingdom.

${ }^{2}$ Royal Veterinary College, Royal College Street, London, NW1 OTU, United Kingdom.

${ }^{3}$ Department of Conservation, Conservation House - Whare Kaupapa Atawhai, PO Box 10420, Wellington 6143, New Zealand.

${ }^{4}$ New Zealand Centre for Conservation Medicine, Auckland Zoo, Private Bag, Grey Lynn, Auckland 1245, New Zealand.

Correspondence: Antonia Dalziel email: antonia.dalziel@adelaide.edu.au

Word count: 4298

Running head: DRA tools for conservation translocations 


\section{Abstract}

Conservation translocations are increasingly used to manage threatened species and restore ecosystems. Translocations increase the risk of disease outbreaks in the translocated and recipient populations. Qualitative disease risk analyses have been used as a means of assessing the magnitude of any effect of disease and the probability of the disease occurring associated with a translocation. Currently multiple alternative qualitative disease risk analysis packages are available to practitioners. Here we compare the ease of use, expertise required, transparency, and results from, three different qualitative disease risk analyses using a translocation of the endangered New Zealand passerine, the hihi (Notiomystis cincta), as a model. We show that the three methods use fundamentally different approaches to define hazards. Different methods are used to produce estimations of the risk from disease, and the estimations are different for the same hazards. Transparency of the process varies between methods from no referencing, or explanations of evidence to justify decisions, through to full documentation of resources, decisions and assumptions made. Evidence to support decisions on estimation of risk from disease is important, to enable knowledge acquired in the future, for example from translocation outcome, to be used to improve the risk estimation for future translocations. Information documenting each disease risk analysis differs along with variation in emphasis of the questions asked within each package. The expertise required to commence a disease risk analysis varies and an action flow chartecision trees tailored for the non-wildlife health specialist are included in one method but completion of the disease risk analysis requires wildlife health specialists with epidemiological and pathological knowledge in all three methods. We show that disease risk analysis package choice may play a greater role in the overall risk estimation of the effect of disease on animal populations involved in a translocation than might previously have been realised.

Keywords: wildlife disease, wildlife health, translocation, reintroduction, hihi, Notiomystis cincta 



\section{Introduction}

Extinction rates and biodiversity loss over the past century have led to an increasing need for management of imperilled species (Kock, Woodford et al. 2010). One method which practitioners utilise is the translocation of animals and plants for conservation purposes. The theory and practice that encompass conservation translocations is now represented as the growing research field of reintroduction biology (Seddon, Armstrong et al. 2007, Ewen, Armstrong et al. 2012) and $\underline{\text { conservation translocations include reintroduction-(IUCN/SSC 1987) [E1], reinforcement, ecological }}$ replacement (Seddon, Armstrong et al. 2007) and assisted colonisation (IUCN/SSC 2013)(Ricciardi and Simberloff 2009).

In any translocation there is inevitably a risk of infectious agent incursion_(Leighton 2002, Kock, Woodford et al. 2010)-. Indeed, translocation of any animal or plant does not constitute the individual alone, but is instead the movement of a complete biological package (Davidson and Nettles 1992), consisting of the host and all of its associated parasites. Disease associated with translocations can occur because (i) parasites (carrier hazards) carried by, or latently infecting, translocated animals cause disease precipitated by stress-induced depression of immunity (ii) parasites, novel to the source and released in the destination, (source hazards) cause disease in species at the destination, (iii) parasites already present in the release environment (destination and population hazards) cause disease in the translocated animals, and-/-or (iv) the translocated animals contract a parasite during transit (transport hazard) which causes disease in translocated or recipient populations (Sainsbury and Vaughan-Higgins 2012). Disease emergence can compromise the survival of translocated and recipient populations and affect the establishment of the released animals (reviewed in (Ewen, Armstrong et al. 2012)). 
Given the potential effect of disease on translocated animals and recipient populations in conservation programmes, efforts have been made to assess this risk and mitigate it (Miller 2007, CBSG 2008, Sainsbury and Vaughan-Higgins 2012). Disease risk analysis (DRA) is one available tool to help mitigate against disease and is a systematic process for identifying the hazards, assessing the magnitude of the potential effect of the disease, the probability that the disease will occur, proposing mitigation measures and communicating the results of the analysis to all stakeholders (Murray, MacDiarmid et al. 2004). Covello \& Merkhofer (1993) were instrumental in the development of a language for risk assessment which could be applied to any situation, dividing the process of risk analysis into three sections; hazard identification, risk assessment and risk evaluation. This language was applied to the risk of disease presented by the movement of domesticated animals by the OIE (World Organization for Animal Health) (Murray, MacDiarmid et al. 2004) using qualitative and quantitative approaches. Recently there have been several attempts to assess the risk of disease in wild animal movements using various modified forms of Covello \& Merkhofer's (1993) DRA (reviewed in Sainsbury et al. 2012)(Sainsbury, Armstrong et al. 2012). All DRAs have the same underlying objective: to identify disease hazards, assess the magnitude and probability of disease occurring, and propose methods to mitigate the risk associated with an animal (or plant) movement. A qualitative method has become the most commonly used form of disease risk analysis in wildlife programs. This is probably because, in most cases, there are simply not theobtaining data available-for quantitative analysis can be difficult[E2] (Leighton 2002), and the number of potential hazards may be very large (Sainsbury, Armstrong et al. 2012), and more quantitative approaches require additional skill sets.

A key feature promoted by the developers of all wild animal DRAs is transparency (Davidson and Nettles 1992, Leighton 2002, Armstrong, Jakob-Hoff et al. 2003, Travis, Hungerford et al. 2006, Miller 2007, Forde-Folle, Mitchell et al. 2011, Sainsbury, Armstrong et al. 2012, Sainsbury and

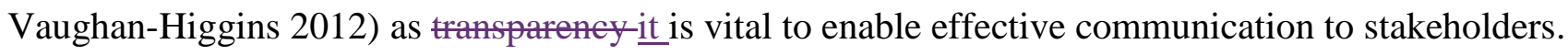


Transparency in DRA is the documenting of all the components used in achieving a risk estimation, including assumptions, methods, results, discussions and conclusions (Murray, MacDiarmid et al. 2004). Without transparency, particularly in a qualitative method, it is difficult to ensure fairness, rationality or consistency in the process of DRA (Murray, MacDiarmid et al. 2004, Travis, Hungerford et al. 2006, Miller 2007).

In spite of a growing number of available DRA methods and their application to conservation translocations (Jakob-Hoff 2001, Sainsbury and Vaughan-Higgins 2012), there have been no studies which have attempted-to compare them, their ease of use, relative transparency, the expertise required, and the risk estimates they produce. Here we directly compare the three most recently used DRA methods using a translocation of a threatened New Zealand bird as a model. Our aims wasere to assess; (i) how concordant the DRA methods were in their risk estimation? (ii) what differences there were in the expertise required? (iii) how easily could each DRA method could be used? (iv) the information and evidence used to explain decisions (relative transparency) in each DRA method, (v) how were-hazards were defined in each method? and (vi) the clarity in defining risk in each DRA method.

\section{Methods}

\section{DRA Methods}

The Conservation Breeding Specialist Group (CBSG) convened in 2003 to develop disease risk assessments for wildlife movements, and published the Animal Movements Disease Risk Workbook (Armstrong, Jakob-Hoff et al. 2003) . Within the CBSG publication was a method developed by one of us (RJ-H) (section 6 workbook), herein referred to as the CBSG method. Of all the methods published by Armstrong et al (2003), this CBSG method most closely follows the DRA method from 
Covello \& Merkhofer (1993). It has been used numerous times for assessing the risk of disease in animal translocations (e.g. Tasmanian devils (Sarcophilus harisii) (CBSG 2008), African lions (Panthera leo) (Michel, Bengis et al. 2006, Keet, Davies-Mostert et al. 2009), amphibians (Zippel, Lacy et al. 2006). Diseases or infectious agents to assess are identified on the basis that they have caused health problems in the target species in the past. The CBSG method asks five questions about each agent, with risk levels then assigned ranging from low to high, and an overall classification of each agent as a hazard of concern or not. For a detailed description of this method see Armstrong et al. (2003).

Masters \& Sainsbury (2011) and Sainsbury \& Vaughan-Higgins (2012) described a modified version of the DRA method used by Murray et al. (2004), for wildlife translocations, hereafter referred to as the IoZ method. This method has been applied to six of the most recent animal conservation translocations in the United Kingdom since 2007. The following are differences from the CBSG method, (i) a parasite need not be known to harm the translocated species to be designated a hazard because observed evidence shows that many disease outbreaks associated with translocations are caused by previously unknown parasites, (ii) a parasite is classified as a hazard if the source and destination populations are separated by ecological or geographical barriers, and the parasite is present only on one side of the barrier, because the parasite may act as a novel pathogen in either translocated or recipient populations, (iii) the effect of stress on host-parasite interactions is evaluated, (iv) hazards thich that occur throughout the translocation pathway are included, and (v) hazards, of potential impact on population numbers, at the destination (population hazards) are considered. The IoZ method considers a specified translocation pathway from source site, through holding and movement, to release at the destination site for each potential hazard (parasite or noninfectious disease agent), justifies hazard status, and, if considered a hazard, then classifies each as a specific hazard type. For a detailed description of this method see Sainsbury \& Vaughan-Higgins (2012). 
The disease risk analysis tool (DRA-T) is a new action flow chart decision tree tool and disease risk workbook (DRW) developed by the Department of Conservation (DOC) in New Zealand (McInnes et al. unpublished). DRA-T has been adopted as the standard DRA protocol for all New Zealand translocations. Consisting of two parts, it aims to streamline the process of approval for wildlife movements. Part A is an action flow chart decision tree-asking twelve questions about the move, which helps the user decide whether there is a need for a more in-depth risk assessment and allows low risk moves, requiring only minimal health measures, to proceed more quickly. Part B, the disease risk workbook (DRW) is based on the CBSG workbook, to be used with the veterinarian wildlife health specialist involved in the project to perform a more in-depth assessment, producing a disease risk estimation table. Diseases are identified on the premise that they have been associated with the species in the past. The workbook asks eleven questions about the disease, including likelihood of being present in the transfer animals, and severity of impact of the disease.

\section{Conservation Translocation Case Study}

The hihi (Notiomystis cincta) is a small endemic passerine of New Zealand. Although once found throughout northern New Zealand, it declined following European colonisation to a single remnant population on an off-shore island (Hauturu, 3083 ha) by the 1890s (Taylor, Castro et al. 2005). Hihi are listed as nationally endangered by the DOC (Miskelly, Dowding et al. 2008) and as Vulnerable by the IUCN's red list (BirdlifeInternational 2012). A key method of management is translocation. Multiple translocations have occurred since 1980, with varying levels of success (Ewen, Renwick et al. 2013).

The source population for our case study is the reintroduced population on Tiritiri Matangi Island ( $\left.36^{\circ} 36^{\prime} \mathrm{S}, 174^{\circ} 53^{\prime} \mathrm{E}\right)$. Tiritiri Matangi is a 220 ha island located in the Hauraki Gulf approximately 30km north east of Auckland, New Zealand (Ewen, Parker et al. 2011). The population was founded in 1995 when 38 hihi were translocated to the island from Hauturu and is 
now maintained between 150-200 adult birds by cropping for translocation (Ewen, Parker et al. 2011). The release site for our case study is Bushy Park Forest Reserve ( $\left.39^{\circ} 48^{\prime} \mathrm{S}, 1^{174^{\circ}} 56^{\prime} \mathrm{E}\right)$. Bushy Park is a 98 ha mainland 'island' that is surrounded with a $4.8 \mathrm{~km}$ predator proof fence (Thorne 2007). All exotic mammals except for mice (Mus musculus) have been eradicated from the park. The park is approximately $25 \mathrm{~km}$ north-west of Wanganui in the south west corner of the North Island and is surrounded by cattle and sheep farms. There have been multiple releases of native bird species into the park, including North Island robins (Petroica longipes) in 2001, and tieke or saddleback (Philesturnus carunculatus) in 2006. The Bushy Park Trust released 44 juvenile hihi into Bushy Park in March 2013.

\section{Choice of parasites and other potential hazards to include in each DRA}

We analysed the risk of disease presented by five representative parasites across a broad taxonomic spectrum using each of the three DRA methods (see Table 1 for list of parasites). We selected parasites known to cause disease in hihi, as determined from necropsy reports or from close monitoring of extant populations (Ewen, Armstrong et al. 2012). These included Salmonellae, namely Salmonella enterica serotype Typhimurium DT195 (Ewen, Thorogood et al. 2007) and $\underline{\text { Salmonella saint-paul (unpublished datavan Andel, Jackson et al. 2014[E3]), Aspergillus fumigatus }}$ (Alley, Castro et al. 1999, Cork, Alley et al. 1999), coccidia (Cork, Alley et al. 1999, Ewen, Armstrong et al. 2012), and Plasmodium relictum (Howe, Castro et al. 2012). We also included two parasites (Trichomonas gallinae; avipox virus) which-that have not been reported in hihi.

Salmonellae were considered both at a species level and as a grouped category of parasites. Considering Salmonellae at the species level was possible because information was available on the

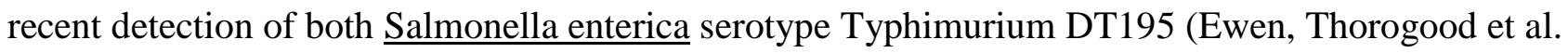
2007) and Salmonella saint-paul (van Andel, Jackson et al. 2014) in hihi on Tiritiri Matangi. We also 
included Salmonella enterica subspecies houtenae because it has been detected in a reptile and one bird species on Tiritiri Matangi_van Andel, Jackson et al. 2014), and Salmonella enterica serotype Typhimurium DT160 because of its presence in passerines in New Zealand (Alley 2002). In addition, a number of deaths and injuries in hihi have been associated with trauma, either from capture or handling (Cork, Alley et al. 1999, Ewen, Armstrong et al. 2012). We therefore deemed trauma an important potential hazard to consider along with infectious parasites.

\section{Method of comparison}

One of us, $\mathrm{AD}$, a qualified wildlife veterinarian, collated available information on the selected potential hazards (Table 1). With assistance from the other authors, and following the published method for each DRA approach, AD undertook a DRA for each parasite, or other potential hazard, using each method. The results were checked and verified by all authors. In no case was a hazard classification altered during this verification phase, indicating that AD performed the DRAs as the authors of each method had intended. We viewed this approach as valid as it; (i) reflected the reality of how these methods are used by conservation translocation practitioners and (ii) was then validated by those who developed each of the DRA tools (the co-authors of this study include those who developed each DRA method). The time and ease of undertaking each DRA method was noted by $\mathrm{AD}$, although the substantial shared information from background research means true quantification between methods was not possible. Instead AD provided a personal opinion to help practitioners judge the relative strengths of alternative approaches. Likewise ${ }_{2}$ the expertise and information required to undertake each method and the clarity of risk definition was noted by AD. Transparency was assessed through (i) the relative amount of documentary evidence recorded, (ii) how the documentary evidence used to inform decisions was acknowledged, (iii) what evidence was provided to support decisions and (iv) how easy it was to check the sources of information used. 


\section{Results}

\section{CBSG Method}

\section{All identified potential hazards (Table 2) were considered with the CBSG method. Risk of disease}

estimates ranged from low to high, and there were two unknowns, $\underline{S}$. typhimurium DT160 and $\underline{T}$. gallinae. Although we, Thoughevaluated all identified-potential hazards (Table 2) were considered with the CBSG method, the-two hazards (name them!) were listed as unknowns were wherebecause for which data available were insufficient to allow evaluation under the CBSG method as [Eu]neither had been seen in hihi or closely related species in New Zealand.**check meaning - the restructure didn't work - I think it now makes sense and fits with our definitions in the methods??*** The probability of disease affecting hihi was predicted to be greatest with the hazards, $\underline{\text { S. typhimurium[E5] }}$

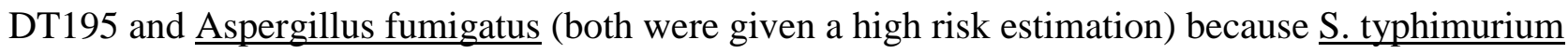
DT195 was present at the source location on Tiritiri Matangi, and highly pathogenic to hihi, and $\underline{\text { A. }}$ fumigatus was ubiquitous in the environment and hihi are susceptible to this pathogen (Perrott and Armstrong 2011) (Table 2). The lack of any positive screening for $\underline{S}$. houtenae in hihi resulted in a 'low' risk estimation despite it being recorded in reptiles at the source site. Low estimated risk of disease for $\underline{\mathrm{S}}$. houtenae was further justified by the relative scarcity of reports of $\underline{\mathrm{S} \text {. houtenae }}$ infecting any bird species in the available global literature.

\section{IoZ Method}

The abbreviated results justifying the status of parasite and other hazards, and defining the hazard

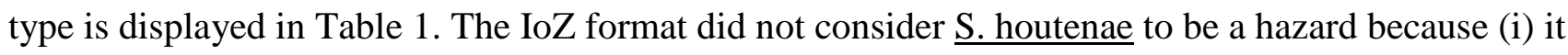
was present at both the source and destination (considering North Island as the destination and 
detection in humans as relevant) sites and therefore was not considered a source or destination hazard, (ii) it has not been detected in faeces of hihi-(Ewen, Thorogood et al. 2007) (Ewen unpublished data) and consequently there is no evidence that hihi are infected and therefore they were not considered a carrier or transport hazard (see Appendix A), (iii) there was no evidence of an impact from this agent at the population level and therefore it was not a population hazard. $-\underline{\mathrm{A}}$. $\underline{\text { fumigatus }}$ was defined as a transport hazard because the ubiquitous spores of $\underline{\mathrm{A} \text {. fumigatus }}$ can remain dormant in the lungs and airsacs of birds (Converse 2007) until the birds are immunosuppressed, such as when stressed during a translocation, and then cause disease (see Box 1). The IoZ method deemed A.fumigatus to be of high risk of precipitating disease in translocated populations.

\section{$D R A-T$}

A detailed DRA was recommended after the second step on the DRA-T decision treeaction flow chart, as the hihi is a DOC listed high priority species. The destination site is also listed as a high priority site, containing populations of nationally critical or nationally endangered species and so a detailed DRA would have, in addition, been recommended at the third step of the decision treeaction flow chart.

The DRW was therefore used to assess the risk for each hazard. The method refers to the risk assessment of disease as a hazard rather than a disease causing agent or parasite. On the basis that the authors of DRA-T intended infectious agents to be considered hazards as in the Covello \& Merkhofer (1993)- method, the highest disease risk estimation (medium-high) was obtained for $\underline{\text { A. }}$

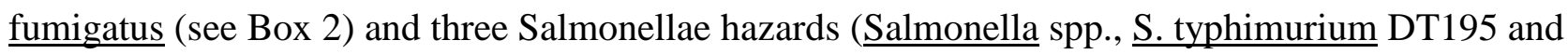

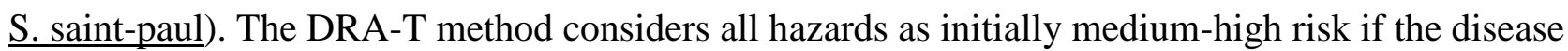
caused by the infectious agent has previously been noted in the species (a risk level whichthat may 
be reduced as a result of carrying out the remainder of the assessment process) and as a result four agents, noted above, were evaluated as medium-high risk. There is no instruction given in the guidelines on how to tally up, or otherwise incorporate, the risk estimates made in response to each question in the decision treeaction flow chart to give an overall risk estimation; however, averaging the risk estimates for all components and giving weight to ability to spread, or cause disease in other species was used (see appendix B) and results were checked by the author of the method. The DRAT did not recognise trauma as a hazard and consequently this hazard was not included.

\section{DRA comparisons}

Each of the three disease risk analysis methods produced different risk estimations for each hazard (Figure 1). The disease risk estimate for the four Salmonella species was inconsistent when compared with estimates of risk of disease at the genus level. For example, the CBSG method generated a low-medium risk for Salmonella ssp., which-it therefore does not qualify as a hazard of

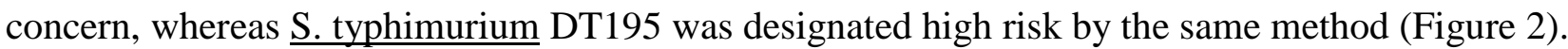
This implies that a potentially high risk hazard might be missed if only a genus level approach was used.

The largest degree of disagreement between the methods is seen with the risk estimation for the avipox viruses, where the estimations range from low to medium-low to high. The three methods differed in their recognition of parasites / non-infectious agents as hazards (Table 1). The CBSG and DRA-T methods defined a parasite as a hazard only if it was a known cause of harm in the target species whilste the IoZ method defined parasites as hazards on the basis of their novelty at the destination, or to the translocated animals, the effects of stress, and on ability to cause population changes at the destination[E6].[E7] The IoZ method discounted one parasite ( $\underline{\text { S houtenae }}$ ) as a hazard because it was present at both the source and destination sites, no carrier state had been identified 
and there was no evidence of population level impacts (Figure 2). Therefore a DRA conducted by a practitioner using the IoZ method dismisses the risk of disease from this type of agent at an early stage.

The IoZ method considers the effects of stressors on parasite pathogenicity because there is evidence that the pathogenicity of infectious agents is increased by stressors during translocation (Dobson and Foufopoulos 2001) and explicitly includes non-infectious agents as hazards. The DRA-T format does not allow for the inclusion of trauma, or other non-infectious diseases, as a hazard even though it has previously been associated with the injury and/or death of hihi.

\section{Discussion}

In this study we compared the relative strengths of three current DRA methods by asking six questions. Firstly, how concordant are the alternative DRA methods in their risk estimation? We found that there was little consistency in estimation of the risk from disease of 10 potential hazards between the three methods. This difference probably stems from differences in the method used to analyse risk. The IoZ method used a release-exposure-consequence assessment approach derived originally from the Covello and Merkhofer (1993) method, as modified by the OIE (Murray, MacDiarmid et al. 2004) which assesses the probability of the release of the hazard, probability of exposure and the probability and magnitude of negative biological, economic and environmental

consequences. CBSG converts a similar approach into an easy-to-complete tabular format which does not specifically guide the assessor to judge the probability and impact of disease. The DRA-T and DRW use a series of questions in an decision treeaction flow chart which are did not aligned to the Covello and Merkhofer (1993) approach and include an assessment of the probability and impact of disease on at risk populations. 
Secondly, what are the differences in the expertise required, for each DRA method? We found that the expertise required to undertake some components of each DRA method varied from minimal, because a simple yes or no answer was required (the decision treeaction flow chart of the DRA-T), to a requirement to provide a full and reasoned justification for inclusion of a given disease causing agent (the justification of hazard status in the IoZ method). The first part of the DRA-T and the majority of the CBSG methods were designed to be used by biologists, and others involved in translocationsreintroduction practitioners, whereas the IoZ method is designed for use by those with knowledge of epidemiology and pathology. For six parasites that we analysed, the DRA-T referred the practitioner at a very early stage to the DRW (because the agents were high risk), and the completion of a DRW required knowledge of epidemiology and pathology. To the best knowledge of the authors, all published cases where DRAs have been used at least one author has been a veterinarian or epidemiologist and advice from those with specialist knowledge is often encouraged (e.g. DRA-T).

Third, how easily could each DRA method be used? Ease of use related largely to the format and evidence required of each method, for example the DRA-T and DRW was an easy and readily usable tick-chart and simple table while-whereas the IoZ method required a complicated, fully referenced and detailed report including detailed evidence to support decisions. The ease of use is fundamentally related to the type and depth of question which is asked by each method that stems, itself, from the original audience for the method. The underlying premise of the DRA-T method is to reduce the delays in approval for low risk translocations, whilst still aiming to prevent diseasethe spread of parasites threats associated with more high risk movements. The IoZ method asks for more information about potential hazards, parasites that may not have been previously identified in the particular species, but could cause detrimental effects, as well as more in depth information about known hazards. 
-Fourth, what is the relative transparency of each of the DRA methods? DRA-T was the least transparent with no requirement for referencing source information or explanation of evidence for decisions made. The CBSG method provided a bibliography of information sources but these were not directly linked to where they were relevant within the process and the evidence for decisions was not explained. The IoZ approach was most transparent by providing in-text citation and an explanation and justification for decisions. Different levels of transparency may be warranted in different circumstances[TS8], and the referencing required is also a function of their utility, reducing the time taken to formalise the evidence but not negating the need to undertake research and investigation into the hazard [Eg]|[TS10]

Fifth, how were the hazards defined? There was a fundamental difference between methods because the IoZ method defined hazards partly on the basis of novelty to the translocated animals or the destination site without knowledge of their pathogenicity. There is strong evidence that the infectious agents of greatest risk of disease to translocations are novel agents or those where the pathogenicity is influenced by stressors (Dobson and Foufopoulos 2001). Several novel infectious agents introduced to naïve populations as a consequence of translocations, whose identity was unknown at the time of translocation, have led to eatastrophic significant mortality outbreaksevents, for example squirrelpox virus (Sainsbury, Deaville et al. 2008) and Batrachochytrium dendrobatidis (Walker, Bosch et al. 2008).[E11] The[TS12] risk of disease outbreaks from such novel agents was used by Sainsbury and Vaughan-Higgins (2012) to justify considering novel parasites as hazards even if they were not known causes of harm. In contrast, DRA-T and CBSG require parasites to be known causes of harm to be considered hazards highlighting underlying differences in the questions being posed by the different methods. The CBSG and DRA T methods do not accommodate novel parasites, and have differing degrees of inquiry between them, whilst the Io $Z$ method defined different parasites as hazards due to potential harm[tS13]-Hon-specialist practitionersmaybe|[E14]_whe has a broader education in disease in multiple species. [TS15]-In addition, if a population of free-living 
animals, in which disease has not been detected, is moved a short distance within New Zealand to a low ecological value DOC site, DRA-T allows this move with 'minimal requirements and recommendations' although such a move could introduce locally novel parasites to the destination with the potential to cause disease.

Sixth, what is the clarity in defining a level of risk for each agent in each DRA method? We found this an inherently difficult area to investigate due to the nature of qualitative assessment and assignment of risk. We note, however, that none of the methods give any direct guidelines of what a low, medium or high risk actually constitutes, although the IoZ method refers to Murray et al (2004), who cited Concise Oxford Dictionary definitions for these parameters.

The lack of concordance between DRA methods in their estimation of risk is perhaps not surprising given it is a qualitative system and ithowever, it must also be noted that each method is asking a different set of questions of a different set of users, with a range of considerations behind their utilisation for multiple types of lapplications][E16][TS17]. We can also not ignore the single translocation project used in this study, and whilst we do draw some conclusions about the methods, we acknowledge the need to repeat the comparison on multiple translocations with different species, including other taxa to ensure the conclusions are applicable to a range of situatiolt would be interesting to repeat these comparisons in other scenarios to test whether similar patterns emerge[E18].*** I really would like this sentence deleted. See comment but regardless it seems off topic and if engaged needs much more thorough treatment easiest just to remove? The desire to look at risk in a quantitative manner is understandable as it divides information into manageable parts that can be assigned a value, but qualitative methods often give a better account of relationships and realities that cannot yet be quantitatively measured (Sofaer 1999).[E19] ****again I really disagree with the message within the now deleted paragraph essential not to quantitatively assess risk!? I think this is sending a bad message. Also its getting into space we haven't focussed on in this MS 
about quantitative stuff.***This means that the above paragraph and this following one could be

joined as are of similar theme and now much shorter... OnefE20] approach to quantify risks within a single DRA method, proposed by Miller et al (2007), is to produce a ranking system for each disease eausing agent. Using the paired ranking of each agent or hazard can allow a hierarchy of risk of disease to be developed for each situation (Miller 2007). However, where uncertainty in knowledge about any two disease causing agents differs this may affect the derived risk estimation, such that knowledge and judgments are frequently not standardized and therefore not directly comparable. It is essential that the qualitative estimations of risk derived in current DRA methods are not given the weight of quantitative evidence, unless uncertainty in these estimates is also captured in the process: Most qualitative risk analyses use a panel of subject experts, and within such a panel questions can be asked about subjective assessments such as, as can be achieved when using a panel of specialist experts and asking for their subjective confidence in their decision, and risk level assignment, though it does require a number of people with specialist knowledge to evaluate appropriately. Following the outcome and analysis of the results of a translocation, the risk level for a succeeding translocation might be revised, but revision is only possible if the evidence for the earlier risk level is transparent. Thus an evidence-based and transparent approach is crucial for the comparison of risk estimations on temporally and spatially separated translocations.

The different information required to make a risk evaluation is likely part of, though not $\underline{\text { solely, }}$ the reason for the different risk estimations obtained by each of the DRA methods. The situationpathway of each translocation may dictate which method can be used, if it is for a move between closely located areas, and there are similar parasite assemblages in each area, limited time and limited information, the DRA-T or CBSG methods may be the best option. If the move is between sensitiveecologically or geographically distant areas, with closely related species that may react very differently to a parasite challenge[TS21] then the IoZ method is likely to be a better option; 
hazards[TS22].

Closely aligned with the information requirements is the level of expertise required by those undertaking a given DRA. The CBSG was originally designed for non-wildlife health specialist[E23]S although it recommends veterinary input for disease related questions. The DRA-T method has also been designed with non-wildlife health specialists in mind, by separating out an initial and simple decision treeaction flow chart component (the DRA-T) that may require the practitioner to seek wildlife health specialist advice under scenarios where the risk is not low (the specialist then uses the DRA-W). Simplification of the process may raise problems, however, if the decisions cannot be robustly justified.

Transparency in decision making and evidence-based conservation is important (Sutherland, Pullin et al. 2004). A recognized problem for $\mathrm{e}$ Current approaches to qualitative DRA is that they often rely on expert judgment (Sainsbury, Armstrong et al. 2012). Expert judgment is important for helping generate alternative hypotheses for how a system operates and how it is expected to respond to management (Gregory, Failing et al. 2012) but is also prone to limiting the range of alternative options considered because of faults with human nature (Burgman 2005). Any approach that more clearly provides the evidence used by experts to make their judgment has the benefit of justification, providing the first steps toward evidence-based decisions, and making uncertainty explicit, such that it may be addressed by future work (for example McBride, Garnett et al., 2012[E24]). Transparency in DRA is rightly given substantial attention in those promoting each method (Murray, MacDiarmid et al. 2004).

Our own comparison of three current and widely used DRA methods has not provided a clear winner. Rather, we have shown that there are benefits and costs of each approach. The relative strengths of each method relate to a trade-off between time available, expertise required and usability 
of the final outcome. Most importantly, however, we can recommend that users familiarise themselves with the requirements of each protocol and are aware of the shortcomings each method contains.

\section{$\underline{\text { References }}$}

Alley, M. (2002). "Wildlife disease surveillance." Surveillance (Wellington) 39(3): 24-26.

Alley, M. R., I. Castro and J. E. Hunter (1999). "Aspergillosis in hihi (Notiomystis cincta) on Mokoia Island." N Z Vet J 47(3): 88-91.

Armstrong, D., R. Jakob-Hoff and U. Seal (2003). "Animal movements and disease risk: a workbook."

Conservation Breeding Specialist Group (Species Survival Commission/IUCN), Apple Valley, USA.

Burgman, M. (2005). Risks and decisions for conservation and environmental management, Cambridge University Press.

CBSG (2008). Tasmanian Devil PHVA final report. Apple Valley, MN.

Converse, K. A. (2007). Aspergillosis. Infections diseases of wild birds. N. J. Thomas, D. B. Hunter and C. T. Atkinson. Ames, Iowa USA, Blackwell Publishing Ltd.: 360-374.

Cork, S. C., M. R. Alley, A. C. Johnstone and P. H. Stockdale (1999). "Aspergillosis and other causes of mortality in the stitchbird in New Zealand." Journal of Wildlife Diseases 35(3): 481-486.

Davidson, W. and V. Nettles (1992). Relocation of wildlife: identifying and evaluating disease risks. Transactions of the North American Wildlife and Natural Resources Conference.

Dobson, A. and J. Foufopoulos (2001). "Emerging infectious pathogens of wildlife." Philosophical Transactions of the Royal Society B: Biological Sciences 356(1411): 1001-1012.

Ewen, J. G., D. P. Armstrong, R. Empson, S. Jack, T. Makan, K. McInnes, K. A. Parker, K. Richardson and M. Alley (2012). "Parasite management in translocations: lessons from a threatened New Zealand bird." Oryx 46(03): 446-456.

Ewen, J. G., D. P. Armstrong and K. A. Parker (2012). Reintroduction biology: integrating science and management, John Wiley \& Sons. 
Ewen, J. G., K. A. Parker, K. Richardson, D. Armstrong and C. Smuts-Kennedy (2011). "Translocation of hihi Notiomystis cincta to Maungatautari, a mainland reserve protected by a predator-exclusion fence, Waikato, New Zealand." Conservation Evidence 8: 58-65.

Ewen, J. G., R. Renwick, L. Adams, D. P. Armstrong and K. A. Parker (2013). 1980 - 2012: 32 years of reintroduction efforts of the hihi (stitchbird) in New Zealand. Global re-introduction perspectives: 2013. Further case-studies from around the globe. P. S. Soorae. Gland, Switzerland, IUCN/SSC Re-introduction Specialist Group and Abu Dhabi, UAE: Environment Agency - Abu Dhabi: 68-73.

Ewen, J. G., R. Thorogood, C. Nicol, D. P. Armstrong and M. Alley (2007). "Salmonella typhimurium in hihi, New Zealand." Emerging Infectious Diseases 13(5): 788.

Forde-Folle, K., D. Mitchell and C. Zepeda (2011). "The role of models in estimating consequences as part of the risk assessment process." Revue scientifique et technique - Office International des Epizooties 30(2). Gregory, R., L. Failing, M. Harstone, G. Long, T. McDaniels and D. Ohlson (2012). Structured decision making: a practical guide to environmental management choices, John Wiley \& Sons.

Howe, L., I. C. Castro, E. R. Schoener, S. Hunter, R. K. Barraclough and M. R. Alley (2012). "Malaria parasites (Plasmodium spp.) infecting introduced, native and endemic New Zealand birds." Parasitology research 110(2): 913-923.

International, B. (2012). Notiomystis cincta. IUCN Red List of Threatened Species Verion 2012.1.

IUCN/SSC (1987). IUCN position statement on translocation of living organisms. Gland, Switzerland, IUCN/SSC.

Jakob-Hoff, R. (2001). Disease risk assessment for translocation of kaki (black stilt), Himantopus novaezelandiae, from captivity to the wild, Department of Conservation.

Keet, D., H. Davies-Mostert, R. Bengis, P. Funston, P. Buss, M. Hofmeyr, S. Ferreira, E. Lane, P. Miller and B. Daly (2009). "Disease risk assessment workshop report: African lion (Panthera leo) Bovine Tuberculosis." Conservation Breeding Specialist Group (CBSG SSC/IUCN)/CBSG Southern Africa. Endangered Wildlife Trust.

Kock, R., M. Woodford and P. Rossiter (2010). "Disease risks associated with the translocation of wildlife." Revue scientifique et technique 29(2): 329.

Leighton, F. (2002). "Health risk assessment of the translocation of wild animals." Revue Scientifique et Technique-Office International des Epizooties 21(1): 187-216.

Michel, A. L., R. G. Bengis, D. Keet, M. Hofmeyr, L. De Klerk, P. C. Cross, A. E. Jolles, D. Cooper, I. Whyte and P. Buss (2006). "Wildlife tuberculosis in South African conservation areas: implications and challenges." Veterinary microbiology 112(2): 91-100.

Miller, P. S. (2007). "Tools and techniques for disease risk assessment in threatened wildlife conservation programmes." International Zoo Yearbook 41(1): 38-51.

Miskelly, C. M., J. E. Dowding, G. P. Elliott, R. A. Hitchmough, R. G. Powlesland, H. A. Robertson, P. M. Sagar, R. P. Scofield and G. A. Taylor (2008). "Conservation status of New Zealand birds, 2008." Notornis 55(3): 117-135. 
Murray, N., S. MacDiarmid, M. Wooldridge, B. Gummow, R. Morley, S. Weber, A. Giovannini and D. Wilson (2004). "Handbook on Import Risk Analysis for Animals and Animal Products-Introduction and Qualitative Risk Analysis, Vol. 1." OIE (World Organisation for Animal Health), Paris.

Perrott, J. K. and D. P. Armstrong (2011). "Aspergillus fumigatus densities in relation to forest succession and edge effects: implications for wildlife health in modified environments." EcoHealth 8(3): 290-300.

Ricciardi, A. and D. Simberloff (2009). "Assisted colonization is not a viable conservation strategy." Trends in ecology \& evolution 24(5): 248-253.

Sainsbury, A. W., D. P. Armstrong and J. G. Ewen (2012). "Methods of disease risk analysis for reintroduction programmes." Reintroduction Biology: integrating science and management 12: 336.

Sainsbury, A. W., R. Deaville, B. Lawson, W. A. Cooley, S. S. Farelly, M. J. Stack, P. Duff, C. J. McInnes, J. Gurnell and P. H. Russell (2008). "Poxviral disease in red squirrels Sciurus vulgaris in the UK: spatial and temporal trends of an emerging threat." EcoHealth 5(3): 305-316.

Sainsbury, A. W. and R. J. Vaughan-Higgins (2012). "Analyzing disease risks associated with translocations." Conserv Biol 26(3): 442-452.

Seddon, P. J., D. P. Armstrong and R. F. Maloney (2007). "Developing the science of reintroduction biology." Conservation Biology 21(2): 303-312.

Sofaer, S. (1999). "Qualitative methods: what are they and why use them?" Health services research 34(5 Pt 2): 1101 .

Sutherland, W. J., A. S. Pullin, P. M. Dolman and T. M. Knight (2004). "The need for evidence-based conservation." Trends Ecol Evol 19(6): 305-308.

Taylor, S., I. C. Castro, R. Griffiths and B. R. Unit (2005). Hihi/stitchbird (Notiomystis Cincta) Recovery Plan, 2004-09, Department of Conservation.

Thorne, J. M. (2007). An Experimental Approach to Translocation of the North Island Saddleback (Philesturnus Carunculatus Rufusater) to Bushy Park Reserve, Wanganui: A Thesis Presented in Partial Fulfilment of the Requirements for the Degree of Masters of Science in Conservation Biology at Massey University, Palmerston North, New Zealand, Massey University, Palmerston North.

Travis, D., L. Hungerford, G. Engel and L. Jones-Engel (2006). "Disease risk analysis: a tool for primate conservation planning and decision making." American journal of primatology 68(9): 855-867.

Walker, S. F., J. Bosch, T. Y. James, A. P. Litvintseva, J. A. O. Valls, S. Piña, G. García, G. A. Rosa, A. A. Cunningham and S. Hole (2008). "Invasive pathogens threaten species recovery programs." Current Biology 18(18): R853-R854.

Zippel, K., R. Lacy and O. Byers (2006). CBSG/WAZA amphibian ex situ conservation planning workshop final report. Apple Valley, MN 55124, USA, IUCN/SSC Conservation Breeding Specialist Group. 
Table 1. Presence or absence of each parasite at the source (Tiritiri Matangi) and destination (North Island) sites for the proposed hihi translocation and the hazard type classification according to the IoZ method. A carrier hazard is a commensal agent which may cause disease in transit or at the destination; a transport hazard is an infectious agent which is acquired by the host during the transportation from source to destination; a population hazard is an infectious or non-infectious agent for which there is evidence of potential effects at the population level at the destination.

Table 2. The results from using the CBSG method for all hazards identified in the hihi translocation case study. Exposure threat ranks the likelihood of exposure and adverse effect. Infectivity ranks the ability for the hazard to infect, survive and multiply in susceptible hosts. Pathogenicity ranks the host-specific ability of the hazard to cause disease. Transmissibility ranks the ability for the hazard to either directly or indirectly transfer from one host to another. Susceptibility ranks the likelihood that the hazard will cause disease in the host. Concern provides a sum of all component rankings, with those reporting a medium or high overall rank being listed as a hazard of concern. Following CBSG protocol a list of references used to justify any ranking are given in a combined reference list (available in Appendix B). Unknown classification indicates that currently information is insufficient for a full assessment to be made. The abbreviation S. Typh stands for Salmonella enterica serotype Typhimurium.

Box 1. An example of a disease risk analysis generated under the IoZ method for the fungal hazard Aspergillus fumigatus

Box 2. An example of an hazard outcome table using the DRW (within DRA-T) for the hazard Aspergillus fumigatus. 
Figure 1. Comparison of the estimated risk of disease for the hazards investigated. Black represents CBSG, light grey represents IoZ and dark grey represents DRA-T .

Figure 2. Comparative risk estimation for Salmonellae overall, and four individual serotypes of Salmonella spp by the three disease risk analysis methods. Black represents CBSG, light grey represents IoZ and dark grey represents DRA-T . 


\section{Aspergillus fumigatus \\ TYPE OF HAZARD}

Carrier

JUSTIFICATION OF HAZARD

Hihi may carry Aspergillus fumigatus spores in airsacs and lungs without symptoms (Converse 2007), but when the host is stressed, as in a translocation, the disease, aspergillosis, can occur often resulting in death (Alley et al. 1999).

\section{RELEASE ASSESSMENT}

Aspergillus fumigatus spores are inhaled and settle out into the airsacs and lungs of hihi, where they can remain dormant, or form fungal plaques (Converse 2007). There is high likelihood of hihi being infected during foraging nesting and roosting in tree cavities, prior to translocation because the fungus is ubiquitous with an almost worldwide distribution (Converse 2007) and favours modified habitats (Perrott \& Armstrong 2011). Tiritiri Matangi is a modified habitat, until the early 1980s it was cleared farmland, since then it has been extensively revegetated and is actively managed (Frost et al. 2012).

\section{EXPOSURE ASSESSMENT}

Hihi may be exposed and develop disease during the quarantine of the translocation process where they will be kept in close quarters with other hihi, as well as being stressed by capture, restraint and sampling procedures. Lowering of host immunocompetence is a factor in development of disease (Converse 2007; Alley et al. 1999). However, only birds which are part of the translocation project are likely to be affected as it is not a contagious disease. A.fumigatus spores are inhaled from the environment. Hihi can be exposed during their normal daily activities, foraging, roosting and nesting in tree cavities. There is high likelihood of the exposure occurring as A.fumigatus is an ubiquitous fungus, and is particularly fond of highly modified environments such as Bushy Park (Perrott $\&$ Armstrong 2011). Aspergillosis is a non-infectious disease with no documented transmission between animals (Converse 2007) and so dissemination is very unlikely. There is a suggestion of a seasonal bias during the winter months for development of aspergillosis in New Zealand (Cork et al. 1999).

\section{CONSEQUENCE ASSESSMENT}

There is high likelihood that at least one hihi will be infected, or may become infected due to the translocation procedure. Aspergillosis is the most commonly diagnosed cause of mortality in hihi [24.4\% in wild birds, 23.6\% wild and captive combined 2001-2008] (Ewen et al. 2012). If it becomes a large scale problem for hihi, there would likely be failure of the translocation, as eventually occurred with the Mokoia Island population (Alley et al. 1999; Armstrong et al. 2007). Apart from failure of translocation, hihi contracting aspergillosis would have little environmental consequence, other than a reduction in ecosystem services that hihi provide as part of the New Zealand native fauna (Kelly et al. 2010). There is low to moderate likelihood of these consequences.

\section{RISK ESTIMATION}

There is high likelihood of hihi being infected when translocated to Bushy Park. There is high likelihood of exposure during quarantine and translocation, however other birds have negligible likelihood of exposure due to the translocation of hihi. There is high likelihood of at least one hihi becoming infected, and low to moderate likelihood of population level consequences. The overall risk estimation is high.

\section{RISK EVALUATION}

The risk estimation is high and sanitary measures are justified.

\section{OPTION EVALUATION}

\section{Practical:}

In a captive situation diagnostic methods for $A$. fumigatus infection includes fungal culture from airsacs and airways, white blood cell count (WBCC), radiographs of respiratory system, biopsy and necropsy. In a field situation only indirect methods such as WBCC may be practical. Post mortem examinations should be carried out on all hihi found dead. Maintaining a clean, dry environment with limited vegetation which may carry spores would reduce the likelihood of greater exposure during quarantine. Current translocation protocol for the hihi includes administration of itraconazole (Sporonox $\left.{ }^{\circledR}\right)(5 \mathrm{mg} / \mathrm{kg}$ body weight/day) from day 3 to release from quarantine(Frost et al. 2012). This should be done, in conjunction with reduction in stress by minimising handling.

Ideal situation:

Spore counts of the permanent quarantine aviaries should be performed annually, possibly at the end of each translocation. To determine the relative differences, if any, between the source and destination sites fungal spore counts should be done at each site (see Perrott \& Armstrong 2011), and management decisions taken in light of those findings. As there is a potential seasonal bias towards the winter months, it may be best to do spore counts in winter.

Alley, M. R., Castro, I., \& Hunter, J. E. (1999). Aspergillosis in hihi (Notiomystis cincta) on Mokoia Island. New Zealand Veterinary Journal, $47(3), 88-91$. doi:10.1080/00480169.1999.36119

Armstrong, D. P., Castro, I., \& Griffiths, R. (2007). Using adaptive management to determine requirements of re-introduced populations: the case of the New Zealand hihi. Journal of Applied Ecology, 44(5), 953-962. doi:10.1111/j.1365-2664.2007.01320.x

Converse, K. A. (2007). Aspergillosis. In N. J. Thomas, D. B. Hunter, \& C. T. Atkinson (Eds.), Infectious Diseases of Wild Birds (1st ed., pp. 360-374). Ames, lowa USA: Blackwell Publishing Ltd.

Cork, S. C., Alley, M. R., Johnstone, a C., \& Stockdale, P. H. (1999). Aspergillosis and other causes of mortality in the stitchbird in New Zealand. Journal of Wildlife Diseases, 35(3), 481-6. Retrieved from http://www.ncbi.nlm.nih.gov/pubmed/10479082

Ewen, J. G., Armstrong, D. P., Empson, R., Jack, S., Makan, T., Mclnnes, K., Parker, K. A., et al. (2012). Parasite management in translocations: lessons from a threatened New Zealand bird. Oryx, FirstView, 1-11. Retrieved from href="http://dx.doi.org/10.1017/S0030605311001281

Frost, P., Ewen, J. G., Armstrong, D. P., Richardson, K., \& Oates, K. (2012). Bushy Park Hihi Translocation Proposal 2012. Wellington, NZ.

Kelly, D., Ladley, J. J., Robertson, A. W., Anderson, S. H., Wotton, D. M., \& Wiser, S. K. (2010). Mutualisms with the wreckage of an avifauna: the status of bird pollination and fruit-dispersal in New Zealand. New Zealand Journal Of Ecology, 34(1), 66-85.

Perrott, J. K., \& Armstrong, D. P. (2011). Aspergillus fumigatus densities in relation to forest succession and edge effects: implications for wildlife health in modified environments. EcoHealth, 8(3), 290-300. doi:10.1007/s10393-011-0716-8 


\section{Box 1. (previous page)}

\section{Box 2.}

Decision tree result: Non-captive population, high priority site. Designated HIGH RISK translocation. Use of workbook required. Disease workbook result:

Disease agent: Aspergillus fumigatus

Disease of concern?: Yes

Zoonosis?: Yes

\begin{tabular}{|l|l|}
\hline Disease name & Aspergillosis \\
\hline $\begin{array}{l}\text { Probability of disease being present } \\
\text { in the transfer animals }\end{array}$ & High \\
\hline $\begin{array}{l}\text { Probability of the disease being } \\
\text { present at the release site }\end{array}$ & Medium \\
\hline $\begin{array}{l}\text { Severity of impact on the transfer } \\
\text { animals if they contract it at the } \\
\text { release site }\end{array}$ & Medium-High \\
\hline $\begin{array}{l}\text { Severity of impact on the } \\
\text { populations at release site if it is } \\
\text { spread by transfer animals }\end{array}$ & Medium-high \\
\hline Ease of spread & Medium-high \\
\hline Rate of spread within the species & Low \\
\hline $\begin{array}{l}\text { Rate of spread between different } \\
\text { species }\end{array}$ & Low \\
\hline Importance of transfer animals & Medium \\
\hline Importance of release site & High \\
\hline Risk of natural spread of the disease & Low \\
\hline Estimated Disease Risk & Medium-high \\
\hline
\end{tabular}


Table 1.

\begin{tabular}{|c|c|c|c|}
\hline $\begin{array}{l}\text { Parasite or potential } \\
\text { hazard }\end{array}$ & Tiritiri Matangi & North & Hazard status and type \\
\hline
\end{tabular}

\begin{tabular}{lccl}
\hline Avian poxvirus & $\checkmark$ & $\checkmark$ & Carrier \\
\hline Salmonella spp. all & $\checkmark$ & $\checkmark$ & Carrier \\
S. typhimurium DT160 & $\times$ & $\checkmark$ & Destination \\
S. typhimurium DT195 & $\checkmark$ & $\checkmark$ & Population \\
S. saint-paul & $\checkmark$ & $\checkmark$ & Carrier \\
S. typhimurium houtenae & $\checkmark$ & $\checkmark$ & Not a hazard \\
Plasmodium relictum & $\checkmark$ & $\checkmark$ & Carrier \\
Coccidia & $\checkmark$ & $\checkmark$ & Carrier \\
Trichomonas gallinae & $\times$ & $\checkmark$ & Destination \\
Aspergillus fumigatus & $\checkmark$ & $\checkmark$ & Carrier \\
Trauma & $\checkmark$ & & Transport / destination \\
& & &
\end{tabular}


Table 2.

\begin{tabular}{|c|c|c|c|c|c|c|c|}
\hline Hazard & Exposure threat & Infectivity & Pathogenicity & Transmissibility & Susceptibility & Concern & Reference \\
\hline S. typh. DT195 & Medium & High & High & Medium-high & High & $\mathrm{Y}$ & $1,2,4,5$ \\
\hline$S$. houtenae & Low & Low & Low & Low & Low & $\mathrm{N}$ & $1,2,5$ \\
\hline Pox avium & Medium & High & Medium & High & High & $\mathrm{Y}$ & $6-8$ \\
\hline Plasmodium relictum & Low & Medium & Medium & Medium & Medium & $\mathrm{Y}$ & $9-13$ \\
\hline Coccidia & Low (effect) & & & & & & \\
\hline Trauma & Medium & - & Medium & - & Medium & $\mathrm{Y}$ & $5,9,24$ \\
\hline
\end{tabular}




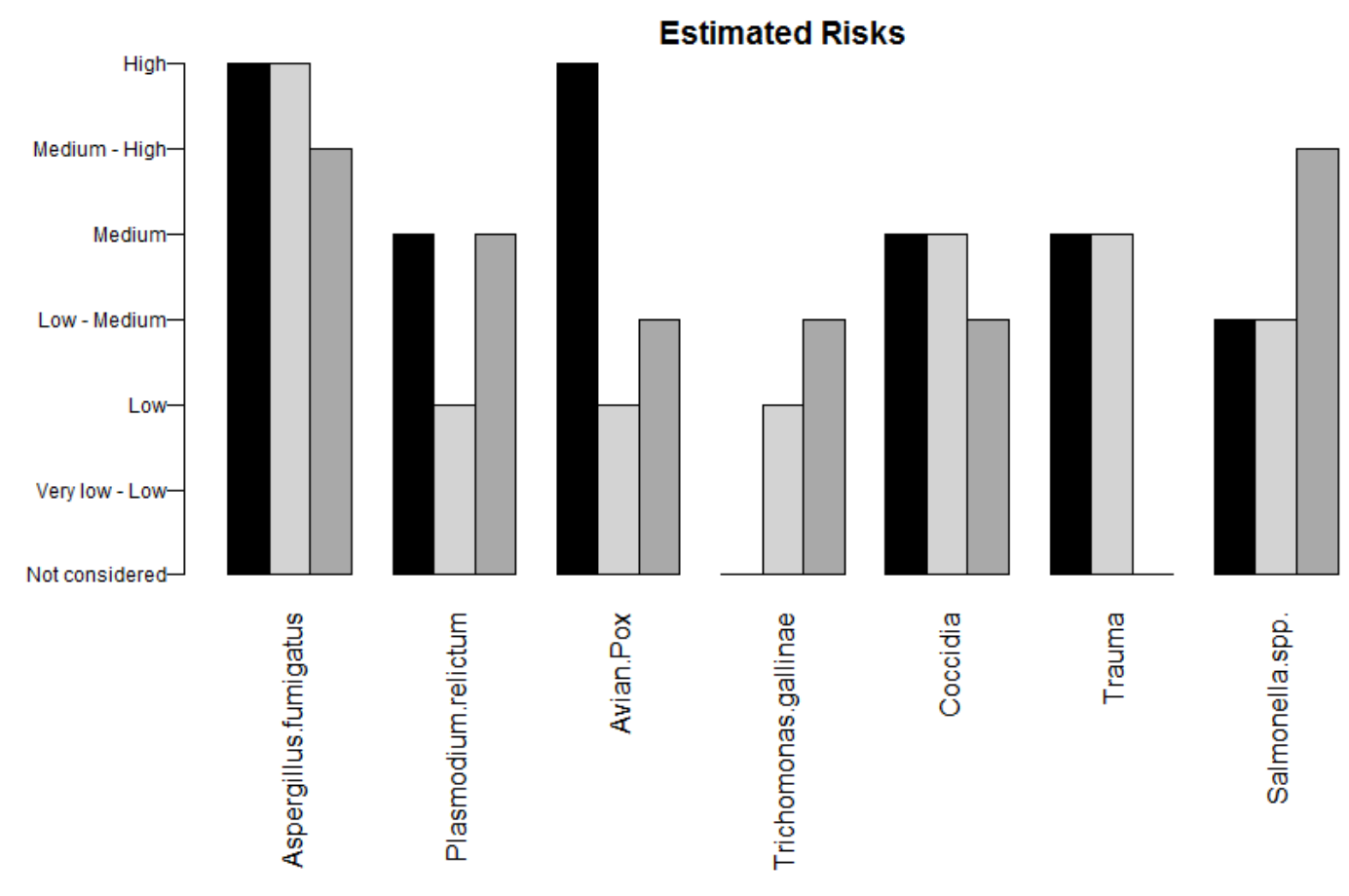

Estimated Risks
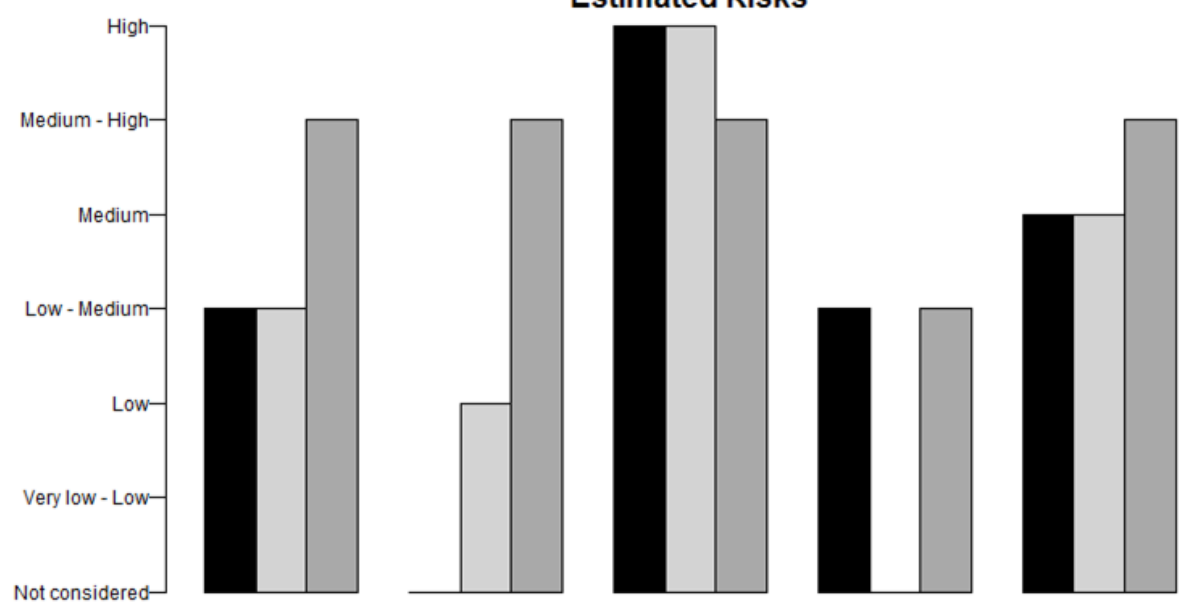

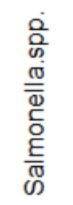
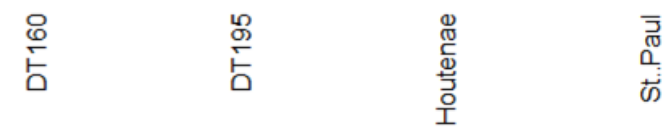